\title{
Tensionless contact of a finite beam resting on Reissner foundation
}

\author{
Yin Zhang* \\ State Key Laboratory of Nonlinear Mechanics (LNM), Institute of Mechanics, Chinese Academy of Sciences, Beijing 100080, China
}

\section{A R T I C L E I N F O}

\section{Article history:}

Received 8 January 2007

Received in revised form

2 February 2008

Accepted 16 February 2008

Available online 23 February 2008

Keywords:

Tensionless contact

Beam

Reissner foundation

Lift-off

\begin{abstract}
A B S T R A C T
A more generalized model of a beam resting on a tensionless Reissner foundation is presented. Compared with the Winkler foundation model, the Reissner foundation model is a much improved one. In the Winkler foundation model, there is no shear stress inside the foundation layer and the foundation is assumed to consist of closely spaced, independent springs. The presence of shear stress inside Reissner foundation makes the springs no longer independent and the foundation to deform as a whole. Mathematically, the governing equation of a beam on Reissner foundation is sixth order differential equation compared with fourth order of Winkler one. Because of this order change of the governing equation, new boundary conditions are needed and related discussion is presented. The presence of the shear stress inside the tensionless Reissner foundation together with the unknown feature of contact area/length makes the problem much more difficult than that of Winkler foundation. In the model presented here, the effects of beam dimension, gap distance, loading asymmetry and foundation shear stress on the contact length are all incorporated and studied. As the beam length increases, the results of a finite beam with zero gap distance converge asymptotically to those obtained by the previous model for an infinitely long beam.
\end{abstract}

(c) 2008 Elsevier Ltd. All rights reserved.

\section{Introduction}

As noted by Timoshenko and Woinowsky-Krieger [1], the corners of a laterally loaded, simply supported rectangular plate in general have the tendency of separating from its supports and the separating of the structure from the support is often called liftingoff [2-10]. The support of Timoshenko and Woinowsky-Krieger is assumed to react to both tension and compression [1]. While, such assumption is motivated more by the desire for mathematical simplicity than by physical reality [4]. The support material is often a rather complex medium which may react only to compression [11] and is thus often referred to as tensionless foundation $[3,9,12]$ or unilateral support $[13,14]$. The contact between the structure and the tensionless support with lifting-off is often named as unbonded contact $[2,5,15]$, which emphasizes the property of lifting-off; or as tensionless contact $[3,6,7,9,10]$, which emphasizes the property of the unilateral response of the support. There are two ways of formulating the tensionless/ unbonded contact problem: integral formulation $[2,6,7,9,16]$ and differential formulation [3,10,12-15]. If the support is modeled as an elastic half-space, in essence it is to solve a Boussinesq problem and its formulation can only be integral $[2,16]$. If the support is modeled as an elastic foundation, its formulation can be either

\footnotetext{
* Tel.: +861062648720 .

E-mail address: zhangyin@Inm.imech.ac.cn
}

integral $[6,7,9]$ or differential $[3,10,12-15]$. Compared with the differential formulation, the integral formulation and the iterative algorithm needed to solve the problem are rather lengthyand complex. On the modeling aspect, the elastic half-space model is also a mathematically much more difficult elasticity problem than the elastic foundation one [11].

The elastic foundation model simplifies the elastic continuum problem by assuming certain relationship between the support reaction/pressure to its surface displacement. For example, the Winkler foundation model is to treat the support as if it consists of closely spaced, independent springs and its pressure is directly related with the spring elongation/compression. However, such assumption may sometimes cause the erroneous results, especially on the stresses inside the elastic continuum. A vivid example given by Johnson [17] demonstrates that the contact pressure profile of a sphere derived from the elastic half-space model and the Winkler foundation model is ellipsoidal and paraboloidal, respectively. Li and Dempsey [5], Akbarov and Kocatürk [8] did the comparative study on the tensionless contact of a plate with a support which is modeled as the Winkler foundation and the elastic half-space, respectively and the results obtained from these two modelings are significantly different. Different elastic foundation models such as Filonenko-Borodich, Hetényi, Pasternak, Vlasov, Reissner foundation models are developed as an effort to better capture the characteristics of an elastic continuum. For the characteristics and development of different elastic foundation models, reader should refer to Kerr's 
papers [11,18] for details. Among those models, Vlasov [19] and Reissner [20] models are the ones of approaching the problem from a continuum point of view. In spite of its conceptual elegance, Vlasov model faces a difficulty in determining an unknown parameter controlling the decay of stress inside the foundation, which results in complex iterative computation [21]. Reissner starts his model by examining the governing equations and boundary conditions of an elastic half-space and obtains relatively simple (as compared with elastic halfspace model) pressure-surface displacement relation by assuming the in-plane (the plane perpendicular to the foundation depth direction) stresses are zero [20]. Reissner's assumption leads to a conclusion that the shear stresses are constant throughout the depth of the foundation [11], which is physically unrealistic, especially for the thick foundation layer. However, "in view of the fact that foundation models are introduced to study the response of the foundation surface to loads and not the stresses caused within the foundation, this particular deficiency may in general be of no serious consequence." [11].

The above studies of tensionless contact [2,4-10,12-16] treat the support either as the Winkler foundation or as an elastic halfspace. Weitsman [3] conducted a comparative study and derived the analytical solutions for an infinitely long beam resting on a tensionless Winkler foundation and Reissner foundation, respectively. However, it has been realized that the boundary conditions used by Weitsman [3] for an infinitely long beam can be problematic for the finite beam/cylinder case $[10,15]$. The nonsymmetric solution terms are thrown away by Weitsman [3] with the assumption that the tensionless contact of an infinitely long beam is symmetric, which as shown later in this paper, is a very reasonable assumption. However, when the beam is finite and loading is asymmetric, this assumption is invalid. Also the gap distance between the structure and support in above studies $[2-9,12-16]$ is zero. In the microelectromechanical systems (MEMS) area, the microstructures contact the substrate due to capillary force, external pressure or electrostatic force and there is a gap distance between the microstructure and the substrate [22-24]. For MEMS devices contact study, it is thus important to incorporate the gap distance as a parameter into the model capable of describing the finite-sized structures. This paper presents a more generalized model on the tensionless contact of a beam on the Reissner foundation, which incorporates the beam dimension, gap distance and loading asymmetry as important parameters in the model. A parameter $\lambda$ which indicates the contribution of shear stress inside the Reissner foundation is introduced and varied to show its influence on the contact. The problem formulation is a differential one which in essence retains the mathematical simplicity of the elastic foundation model.

\section{Equations of equilibrium and its solutions}

As shown in Fig. 1(a), a hinged-hinged beam is separated from the Reissner foundation with the gap distance of $W_{o}$. $E_{f}$ and $G_{f}$ are Young's modulus and shear modulus of the Reissner foundation, respectively. $P$ is the concentrated load and the coordinate system starts at the loading point. $L_{1}$ and $L_{2}$ are the distances from the loading point to the beam left and right ends, respectively. $L=L_{1}+L_{2}$ is the beam length and $E I$ is the beam flexural rigidity. $H$ is the layer thickness of the Reissner foundation. $X_{1}$ is leftside contact length and $X_{2}$ is the rightside contact length ( $X_{1}$ and $X_{2}$ are positive numbers). The beam will separate from the foundation once the beam deflection $W$ is less than the gap distance $W_{o}$. The beam deflection $W$ is divided into

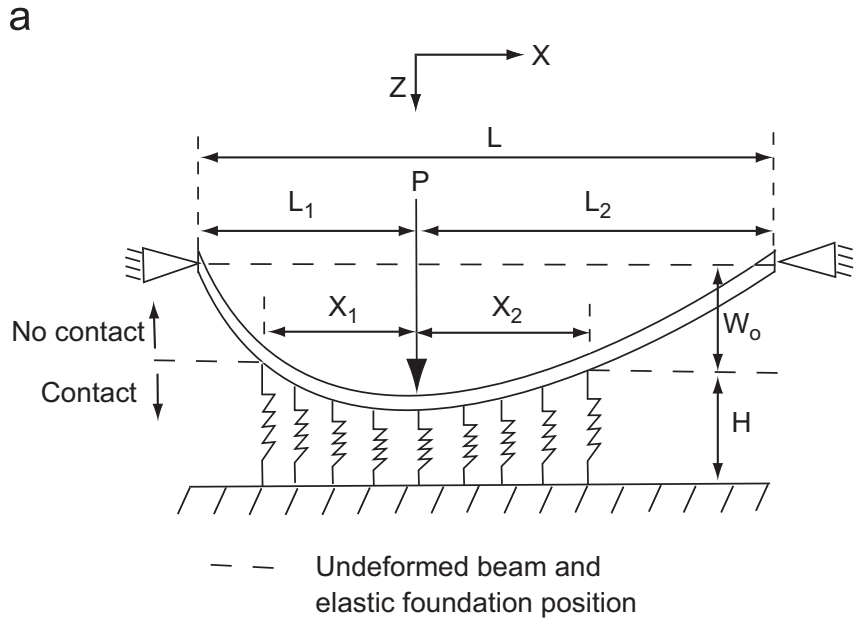

b

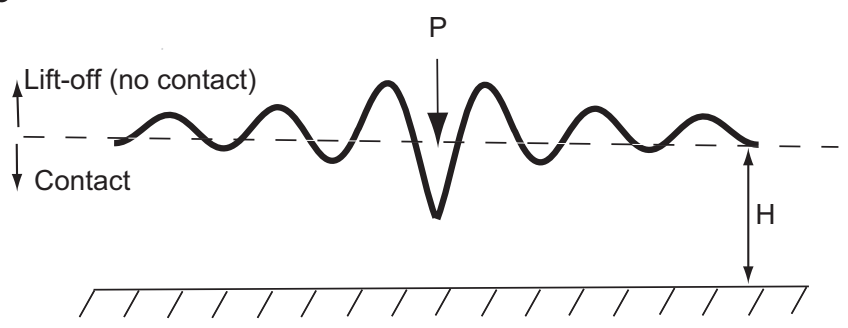

Fig. 1. (a). The schematic diagram and related coordinate system of the continuous contact of a beam under a point load $P$. (b) The discontinuous contact scenario.

the following three regions and the governing equation for each region holds as follows $[3,10]$ :

$$
\left\{\begin{array}{l}
E I \frac{\mathrm{d}^{4} W_{1}}{\mathrm{~d} X^{4}}=0, \quad W_{1}<W_{o}, \quad-L_{1}<X<-X_{1} \\
\frac{E I G_{f} H^{3}}{12 E_{f}^{2}} \frac{\mathrm{d}^{6} V}{\mathrm{~d} X^{6}}-\frac{D H}{E_{f}} \frac{\mathrm{d}^{4} V}{\mathrm{~d} X^{4}}+\frac{G_{f} H^{2}}{3 E_{f}} \frac{\mathrm{d}^{2} V}{\mathrm{~d} X^{2}}-V \\
\quad=-\frac{H}{E_{f}} P \delta(X)+\frac{G_{f} H^{3}}{12 E_{f}^{2}} P \frac{\mathrm{d}^{2} \delta(X)}{\mathrm{d} X^{2}}, \quad W_{2} \geqslant W_{o}, \quad L_{1} \leqslant X \leqslant X_{2} \\
E I \frac{\mathrm{d}^{4} W_{3}}{\mathrm{~d} X^{4}}=0, \quad W_{3}<W_{o}, \quad X_{2}<X<L_{2}
\end{array}\right.
$$

$V=W_{2}-W_{o}$ and $\delta$ is the Dirac delta function. The above governing equation does not account for the beam self weight, which can be modeled as a uniform load [3]. The above equation also implicitly assumes the so-called continuous contact scenario [3]. For the beam with self weight or uniformly distributed downward load, the beam can have the contact scenario as shown in Fig. 1(b). As the contact regions in Fig. 1(b) are separated from one another, the contact scenario is called discontinuous contact [2]. However, the concentrated load required for the discontinuous contact to occur is so large and the beam will collapse at a much smaller concentrated load. Therefore, it is of no practical use to analyze such contact scenario [3]. To nondimensionalize Eq. (1), the following quantities are introduced

$$
\begin{aligned}
& k=\frac{E_{f}}{H}, \quad \beta^{4}=\frac{k}{4 E I}, \quad \xi=\beta X, \\
& w=\beta W, \quad v=\beta V, \quad \lambda^{2}=\frac{H^{2} \beta^{2} G_{f}}{48 E_{f}}
\end{aligned}
$$


With the substitution of $v=w_{2}-w_{0}$, the dimensionless form of Eq. (1) now is as follows:

$$
\left\{\begin{array}{l}
\frac{\mathrm{d}^{4} w_{1}}{\mathrm{~d} \xi^{4}}=0, \quad w_{1}<w_{o}, \quad-l_{1}<\xi \leqslant-\xi_{1} \\
\lambda^{2} \frac{\mathrm{d}^{6} w_{2}}{\mathrm{~d} \xi^{6}}-\frac{1}{4} \frac{\mathrm{d}^{4} w_{2}}{\mathrm{~d} \xi^{4}}+16 \lambda^{2} \frac{\mathrm{d}^{2} w_{2}}{\mathrm{~d} \xi^{2}}-w_{2}+w_{0} \\
=-F \delta(\xi)+4 \lambda^{2} F \delta^{\prime \prime}(\xi), \quad w_{2} \geqslant w_{0}, \quad-\xi_{1} \leqslant \xi<-\xi_{2} \\
\frac{\mathrm{d}^{4} w_{3}}{\mathrm{~d} \xi^{4}}=0, \quad w_{3}<w_{0}, \quad \xi_{2} \leqslant \xi<l_{2}
\end{array}\right.
$$

It is interesting to notice that in the above equation, the dimensionless gap distance $w_{0}$ plays exactly the same role as the beam weight in Weitsman's governing equation [3]. $\xi_{1}, \xi_{2}, l_{1}$, $l_{2}, l, w_{o}, w_{i}(i=1,2,3)$ are defined as

$\xi_{1}=\beta X_{1}, \quad \xi_{2}=\beta X_{2}, \quad l_{1}=\beta L_{1}, \quad l_{2}=\beta L_{2}$,

$l=\beta L, \quad w_{o}=\beta W_{o}, \quad w_{i}=\beta W_{i}(i=1,2,3)$

The characteristic equation of $w_{2}$ in Eq. (3) is as follows:

$\phi^{6}-\frac{1}{4 \lambda^{2}} \phi^{4}+16 \phi^{2}-\frac{1}{\lambda^{2}}=0$

By letting $\Delta=\phi^{2}$, Eq. (5) can be written as a cubic equation

$\Delta^{3}-\frac{1}{4 \lambda^{2}} \Delta^{2}+16 \Delta-\frac{1}{\lambda^{2}}=0$

The three roots of Eq. (6) can be obtained from Cardan solution [25]. In this case, the three roots are always that one is real and the other two are complex conjugates. Therefore, the six roots of Eq. (5) which are the square roots of those three roots of Eq. (6) are obtained as follows:

$\phi_{1,2}= \pm \alpha, \quad \phi_{3,4,5,6}= \pm \eta \pm \gamma \mathrm{i}$

$\alpha, \eta, \gamma$ are three real number. So there are two real roots and four complex conjugate roots for Eq. (5). These six roots give the solution form of homogeneous part of $w_{2}$. The solutions of Eq. (3) are now given as the following three equations:

$w_{1}=B_{1} \xi^{3}+B_{2} \xi^{2}+B_{3} \xi+B_{4}$

$\begin{aligned} w_{2}= & A_{1} \cosh (\alpha \xi)+A_{2} \sinh (\alpha \xi)+A_{3} \sinh (\eta \xi) \sin (\gamma \xi) \\ & +A_{4} \sinh (\eta \xi) \cos (\gamma \xi)+A_{5} \cosh (\eta \xi) \sin (\gamma \xi) \\ & +A_{6} \cosh (\eta \xi) \cos (\gamma \xi)+A \sinh |\alpha \xi| \\ & +B \cosh (\eta \xi) \sin |\gamma \xi|+D \sinh (\eta \xi) \cos |\gamma \xi|+w_{o}\end{aligned}$

$w_{3}=C_{1} \xi^{3}+C_{2} \xi^{2}+C_{3} \xi+C_{4}$

Here $B_{i}, C_{i}(i=1-4)$ and $A_{j}(j=1-6)$ are the unknown constants to be determined. Because the separation points $\left(\xi_{1}\right.$ and $\left.\xi_{2}\right)$ are also unknown, there are total 16 unknowns to be determined. The first six terms in Eq. (9) are the homogeneous solution and the last three terms are the particular solution. $A, B$ and $D$ are the three constants given as follows:

$A=\frac{4 F\left(\gamma^{4}-\eta^{4}\right)}{\alpha \bar{\Delta}}, \quad B=\frac{F\left(\alpha^{4}-\eta^{4}+10 \eta^{2} \gamma^{2}-5 \gamma^{4}\right)}{\gamma \bar{\Delta}}$,

$D=\frac{F\left(-\alpha^{4}+\gamma^{4}-10 \eta^{2} \gamma^{2}+5 \eta^{4}\right)}{\eta \bar{\Delta}}$
With the following definition of $\bar{\Delta}$ as

$\bar{\Delta}=\left(\eta^{2}+\gamma^{2}\right)\left[\alpha^{4}-2 \alpha^{2}\left(\eta^{2}-\gamma^{2}\right)+\left(\eta^{2}+\gamma^{2}\right)^{2}\right]$

Here it would be instructive to have a comparison with Weitsman's formulation/solution on this problem. Geometrically, Weitsman's beam length is infinite and the gap distance is zero $\left(w_{o}=0\right)$ [3]. Weitsman's governing equation is only for the contact part and his solution is the following:

$$
\begin{aligned}
w_{2}= & A_{1}^{\prime} \cosh (\alpha \xi)+A_{3}^{\prime} \sinh (\eta \xi) \sin (\gamma \xi)+A_{6}^{\prime} \cosh (\eta \xi) \\
& \times \cos (\gamma \xi)+A \sinh |\alpha \xi|+B \cosh (\eta \xi) \sin |\gamma \xi| \\
& +D \sinh (\eta \xi) \cos |\gamma \xi|
\end{aligned}
$$

$A_{1}^{\prime}, A_{3}^{\prime}$ and $A_{6}^{\prime}$ are three unknown constants to be determined. The reason for the above solution is due to the assumption that the contact area is symmetric to the concentrated load, which is also selected as the coordinate origin. The functions associated with $A_{1}^{\prime}, A_{3}^{\prime}, A_{6}^{\prime}, A, B$ and $D$ are all even functions. Those terms of odd functions are thrown away because of this symmetry assumption. For a beam with finite length and the concentrated load off the center, Weitsman's solution above cannot be valid any more and those terms associated with odd functions survive. However, as discussed later, when the beam length is large, those odd function terms do not contribute much to the solution, in other words, Weitsman's solution is a very good approximation.

\section{Boundary/matching conditions}

As mentioned above, there are total 16 unknowns to be determined $\left(B_{i}, C_{i}(i=1-4), A_{j}(j=1-6)\right.$ and $\left.\xi_{1}, \xi_{2}\right)$. Therefore, 16 boundary/matching conditions are needed for the problem solving.

At $\xi=-\xi_{1}, \xi_{2}$, the beam separates from the elastic foundation and different governing equations apply as reflected from Eq. (1) or Eq. (3). Certain conditions must be satisfied for $w_{1}, w_{2}$ and $w_{3}$ to match each other. The following two equations give the eight matching conditions:

$$
\begin{aligned}
& w_{1}\left(-\xi_{1}\right)=w_{2}\left(-\xi_{1}\right), \quad \frac{\mathrm{d} w_{1}\left(-\xi_{1}\right)}{\mathrm{d} \xi}=\frac{\mathrm{d} w_{2}\left(-\xi_{1}\right)}{\mathrm{d} \xi}, \\
& w_{2}\left(\xi_{2}\right)=w_{3}\left(\xi_{2}\right), \quad \frac{\mathrm{d} w_{2}\left(\xi_{2}\right)}{\mathrm{d} \xi}=\frac{\mathrm{d} w_{3}\left(\xi_{2}\right)}{\mathrm{d} \xi} \\
& \frac{\mathrm{d}^{2} w_{1}\left(-\xi_{1}\right)}{\mathrm{d} \xi^{2}}=\frac{\mathrm{d}^{2} w_{2}\left(-\xi_{1}\right)}{\mathrm{d} \xi^{2}}, \quad \frac{\mathrm{d}^{3} w_{1}\left(-\xi_{1}\right)}{\mathrm{d} \xi^{3}}=\frac{\mathrm{d}^{3} w_{2}\left(-\xi_{1}\right)}{\mathrm{d} \xi^{3}}, \\
& \frac{\mathrm{d}^{2} w_{2}\left(\xi_{2}\right)}{\mathrm{d} \xi^{2}}=\frac{\mathrm{d}^{2} w_{3}\left(\xi_{2}\right)}{\mathrm{d} \xi^{2}}, \quad \frac{\mathrm{d}^{3} w_{2}\left(\xi_{2}\right)}{\mathrm{d} \xi^{3}}=\frac{\mathrm{d}^{3} w_{3}\left(\xi_{2}\right)}{\mathrm{d} \xi^{3}}
\end{aligned}
$$

Eq. (14) is the geometric conditions which require the continuity of the beam deflection and its slope at the separation points. Physically, Eq. (14) plays the role of compatibility condition to guarantee that there is no beam fracture/breaking at the separation points. Eq. (15) is the natural boundary conditions which require the continuity of bending moment and shear force. Also at the separation points, the following two matching conditions hold:

$\frac{\mathrm{d}^{4} w_{2}\left(-\xi_{1}\right)}{\mathrm{d} \xi^{4}}=0, \quad \frac{\mathrm{d}^{4} w_{2}\left(\xi_{2}\right)}{\mathrm{d} \xi^{4}}=0$

At the separation points there is no interaction between the beam and foundation, Eq. (16) physically states that the pressure stress at the separation points is zero [3]. There are two displacement constraint conditions at $\xi=-\xi_{1}, \xi_{2}$

$w_{2}\left(-\xi_{1}\right)=w_{o} \quad\left(\right.$ or $\left.w_{1}\left(-\xi_{1}\right)=w_{0}\right)$,
$w_{2}\left(\xi_{2}\right)=w_{0} \quad\left(\right.$ or $\left.w_{3}\left(\xi_{2}\right)=w_{0}\right)$

Eq. (17) prescribes the separation rule that the beam lifts-off from the tensionless foundation once its deflection is less than the gap 
distance. For a hinged-hinged beam, the boundary conditions at the ends are as follows:

$w_{1}\left(-l_{1}\right)=0, \quad \frac{\mathrm{d}^{2} w_{1}\left(-l_{1}\right)}{\mathrm{d} \xi^{2}}=0$,

$w_{3}\left(l_{2}\right)=0, \quad \frac{\mathrm{d}^{2} w_{3}\left(l_{2}\right)}{\mathrm{d} \xi^{2}}=0$

Eqs. (14)-(18) offer 16 boundary/matching conditions in total. By substituting the solution forms of $w_{1}, w_{2}$ and $w_{3}$ into those 16 boundary/matching conditions, those 16 unknowns can be found. Because of the unknown feature of $\xi_{1}$ and $\xi_{2}$, solving these 16 unknowns is a nonlinear problem and Newton-Raphson method is applied here.

Again, here let us have a discussion on Weitsman's boundary/ matching conditions. For Weitsman's case, there are only four unknowns, $A_{1}^{\prime}, A_{3}^{\prime}, A_{6}^{\prime}$ and $\xi_{1}\left(\xi_{1}=\xi_{2}\right.$ due to the symmetry assumption). Weitsman's four boundary conditions are as follows [3]:

$\frac{\mathrm{d}^{2} w_{2}\left(\xi_{1}\right)}{\mathrm{d} \xi^{2}}=0, \quad \frac{\mathrm{d}^{3} w_{2}\left(\xi_{1}\right)}{\mathrm{d} \xi^{3}}=0, \quad \frac{\mathrm{d}^{4} w_{2}\left(\xi_{1}\right)}{\mathrm{d} \xi^{4}}=0$,

$\frac{G_{f}}{24}\left[\frac{\mathrm{d}^{5} w_{2}\left(\xi_{1}\right)}{\mathrm{d} \xi^{5}}+\frac{16 \mathrm{~d} w_{2}\left(\xi_{1}\right)}{\mathrm{d} \xi}\right]=0$

The first three boundary conditions indicate no bending moment, shear force and pressure stress at the separation point. Compared with Eq. (19), Eq. (15) does not indicate the vanishing of bending moment and shear force at the separation point. Kerr derives the matching conditions of a plate on a Pasternak foundation by a variational approach and his matching conditions are very similar to those in Eqs. (14) and (15), which explicitly negate the vanishing of bending moment and shear force as the matching conditions [15]. The last boundary condition in Eq. (19) indicates that at separation point there is no shear stress. Here it is worth discussing this last boundary conditions. This no shear stress condition at the separation point is originally given by Reissner as a "simple and reasonable condition" without a proof [20]. Kerr's analysis shows that this no shear stress condition "is valid only in the very special case when the foundation material under the finite plate is separated along the cylindrical boundary from the surrounding foundation materials or walls" [11]. The more generalized boundary condition as a substitution for the no shear stress condition given by Kerr is as follows [11]:

$D \nabla^{4} V+\frac{4 E_{f}}{H} V-q=0$

$V=W-W_{o}$ as defined before. $\nabla^{2}=\partial^{2} / \partial X^{2}+\partial^{2} / \partial Y^{2}$ is the Laplacian operator and $\nabla^{4}=\left(\nabla^{2}\right)^{2}$. For one-dimensional case, $\nabla^{4}=\mathrm{d}^{4} / \mathrm{d} X^{4} . D$ is the plate/beam flexural rigidity and $q$ is a uniformly distributed external load. Here the beam is weightless, so $q=0$. Also at $X_{2}$ and $-X_{1}, V=0$. Therefore, the dimensionless boundary condition derived from Eq. (20) is as follows:

$\frac{\mathrm{d}^{4} v}{\mathrm{~d} \xi^{4}}=\frac{\mathrm{d}^{4} w_{2}}{\mathrm{~d} \xi^{4}}=0$

This boundary condition has already been used in Eq. (19). It is noticed that Weitsman's boundary conditions of Eq. (19) start with the second derivative and there is no information for the deflection and slope at the separation point. For the study of the tensionless Winkler foundation, Weitsman prescribes the vanishing of the deflection and slope as the boundary conditions [3]. It is also noticed that when $G_{f}=0$, which is the limit case of Winkler foundation, the fourth boundary condition of no shear stress in Eq. (19) is automatically satisfied. So there is some inconsistency here. Weitsman's formulation focuses only on the contact part and the formulation presented here considers both contact and lifting- off parts. However, in the next section Weitsman's formulation is demonstrated to be an approximation with high accuracy when the beam length is very large.

\section{Results and discussion}

Fig. 2 shows $\lambda$ versus the contact lengths of hinged-hinged beams with different lengths. The gap distance $w_{o}$ is zero. The concentrated load $F$ is symmetric, i.e., at the center of the beam, so the left and right contact lengths are the same $\left(\xi_{1}=\xi_{2}\right)$ due to the symmetry. $\lambda$ is the parameter defined in Eq. (2), which physically indicates the shear stress contribution to the total foundation pressure as compared with the normal one. As mentioned before, the foundation becomes Winkler foundation when $\lambda$ or $G_{f}$ is zero. Fig. 2 shows that the beam with longer length has smaller contact length because longer beam means larger flexurality and more beam parts lift-off from the foundation. The contact length decreases with the increase of $\lambda$ and later converges when $\lambda$ is large enough. For an infinitely long beam with zero gap distance, Weitsman [3] obtains $\xi_{1}=\pi / 2 \approx 1.5708$ when $\lambda=0$ and $\xi_{1}=$ $\pi / 2 \sqrt{2} \approx 1.1107$ as $\lambda \rightarrow \infty$. For the beam of $l=200$ computed here, $\xi_{1}=1.58$ when $\lambda=0$ and $\xi_{1}=1.12$ as $\lambda \rightarrow \infty$. By further increasing the beam length $l$, the contact length asymptotically converges to Weitsman's results. When the beam with finite length and under an asymmetric loading, the left and right contact lengths are in general different from each other, i.e., $\xi_{1} \neq \xi_{2}$. Fig. 3 shows the beam left and right contact lengths under an asymmetric loading at $l_{1}=0.6 l$ and the gap distance is also zero. Clearly from Fig. 3 , the short beam $(l=20)$ is more sensitive to the asymmetric loading and its left and right contact lengths are different from each other. When the beam length increases, the difference between left and right contact lengths shrinks. When $l=200$ and $\lambda=2, \xi_{1}=1.124$ and $\xi_{2}=1.127$; the difference between the left and right contact lengths now is very small. The contact length of the beam under a symmetric loading is $\xi_{1}=$ $\xi_{2}=1.125$ when $l=200$ and $\lambda=2$. This in essence demonstrates that the Weitsman's symmetric assumption for an infinitely long beam as discussed above is an approximation with high accuracy. It is noticed that in both Figs. 2 and 3 of $w_{0}=0$, the contact lengths are independent of the magnitude of $F$. This interesting and somewhat surprising result is due to the lifting-off mechanism, which also occurs in the Winkler foundation $[3,6,9,10]$ and

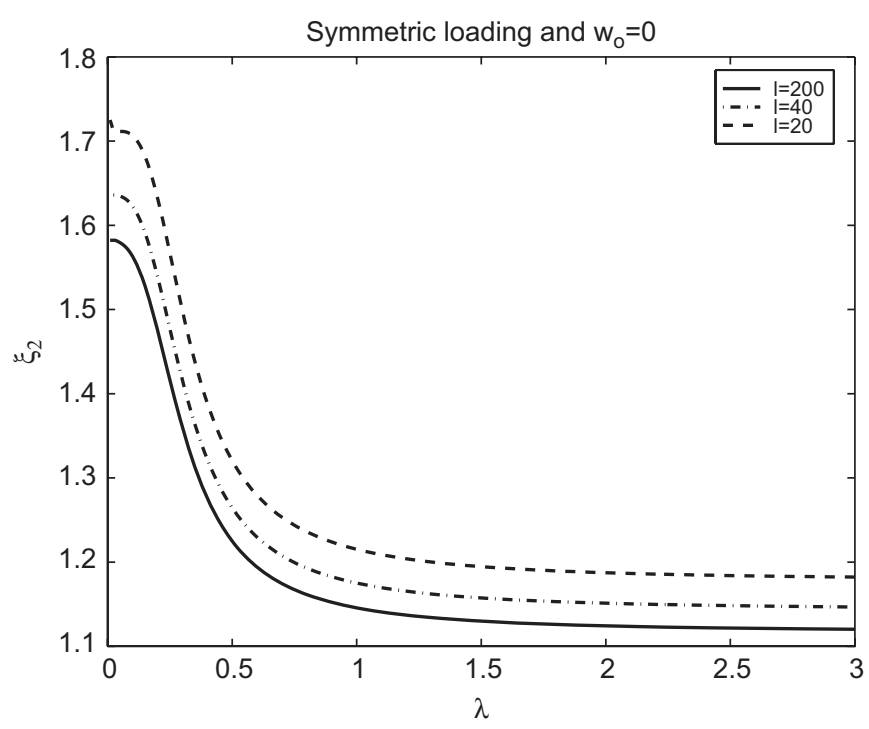

Fig. 2. $\lambda$ versus contact lengths of a hinged-hinged beam with the three different lengths $(l)$ under a symmetric loading $\left(l_{1}=0.5 l\right)$. The gap distance is $w_{o}=0$. 


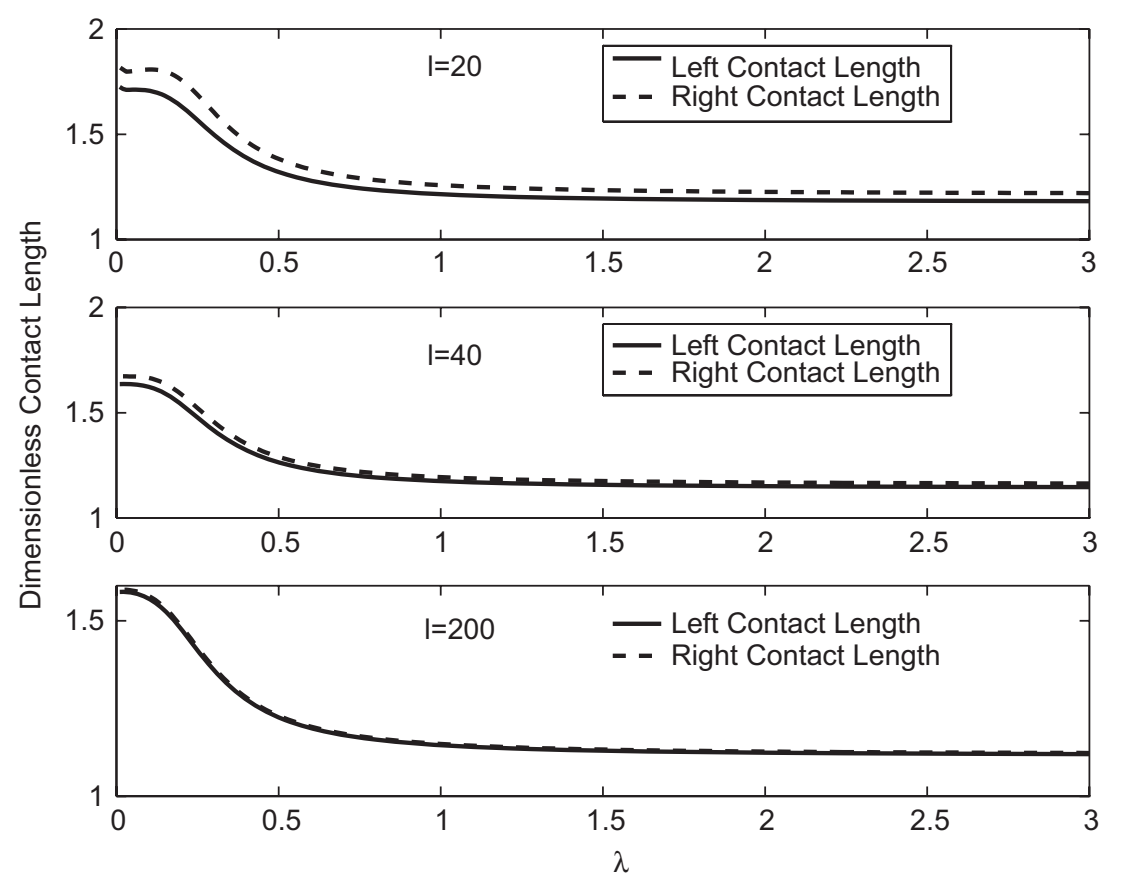

Fig. 3. $\lambda$ versus contact length of a hinged-hinged beam with the three different lengths $(l)$ under an asymmetric loading $\left(l_{1}=0.6 l\right)$. The gap distance is $w_{0}=0$.

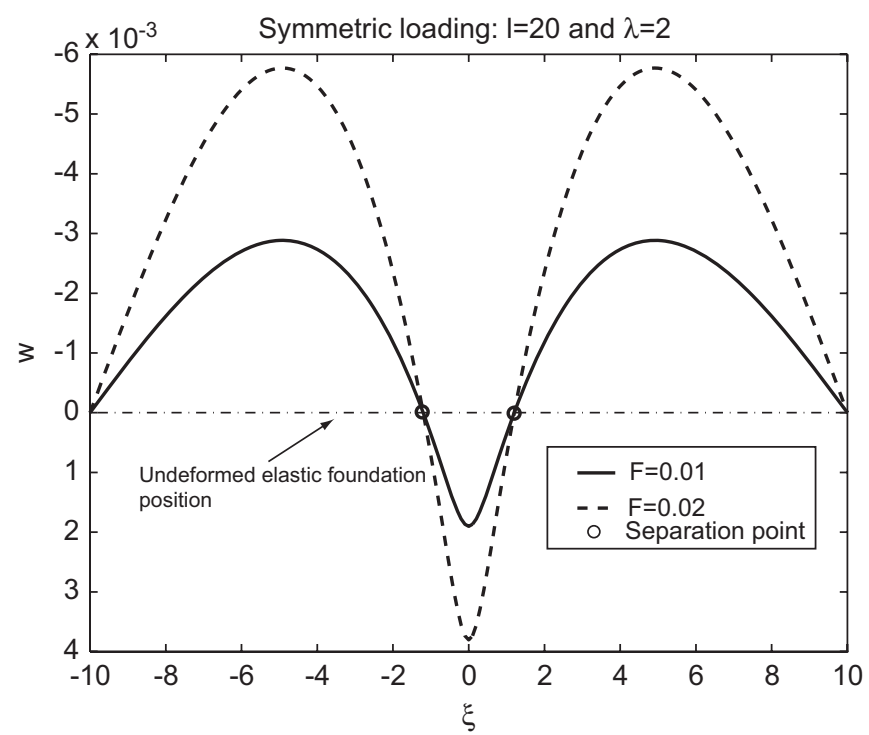

Fig. 4. The deflection of the beam with $l=20$ and $\lambda=2$ under the symmetric loadings of $F=0.01$ and 0.02 , respectively.

elastic half-space cases [2]. To explain this, let us examine the beam deflection under different loads. Fig. 4 plots the beam deflections under two symmetric loadings of $F=0.01$ and 0.02 , respectively. Although the loads are different, clearly form Fig. 4 the beam lifts-off/separates from the elastic foundation at the exactly same points. The larger load $F$ just pushes the beam deeper into the foundation without the change of contact length. Fig. 5 plots the beam deflections under two asymmetric loadings of $F=0.01$ and 0.02 (both located at $l_{1}=0.6 l$ ), respectively. The same scenario happens again that the larger load just pushes the beam deeper into the foundation without changing either the left or right contact length.

Fig. 6 plots the dimensionless contact length of the beam with $l=20$ and $w_{o}=0.1$ under the different symmetric loads of $F$ as a

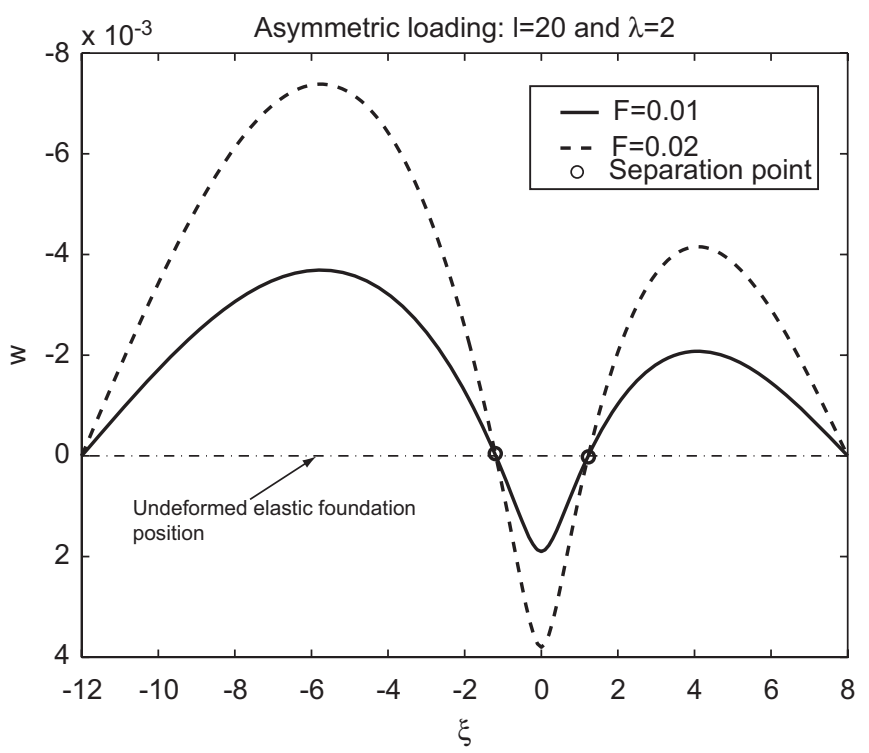

Fig. 5. The beam deflection with $l=20$ and $\lambda=2$ under the asymmetric loadings of $F=0.01$ and 0.02 , respectively. The asymmetric loadings are both located at $l_{1}=0.6 l$.

function of $\lambda$. Unlike those in Figs. 2 and 3, the contact lengths in Fig. 6 are no longer independent on the magnitude of $F$. For a comparison reason, the contact length of the same size beam with $w_{0}=0$ is also plotted in Fig. 6. Fig. 6 shows that larger load $F$ has larger contact length for the beam with $w_{o}=0.1$. It is also noticed that as $F$ increases, the curves of $w_{0}=0.1$ approaches the curve of $w_{o}=0$. The curve of $F=0.4$ and $w_{0}=0.1$ is very close to that of $w_{o}=0$. Again, here it will be instructive for us to examine the beam deflection under different loads when the gap distance is nonzero. Fig. 7 plots the deflections of the beam with $l=20, w_{o}=$ 0.1 under the load $F=0.01$ for $\lambda=0.1$ and 2 , respectively. The contact length is $\xi_{1}=\xi_{2}=1.3293$ when $\lambda=0.1$ and $\xi_{1}=\xi_{2}=$ 0.916 when $\lambda=2$. As a comparison, Fig. 8 plots the deflection of 


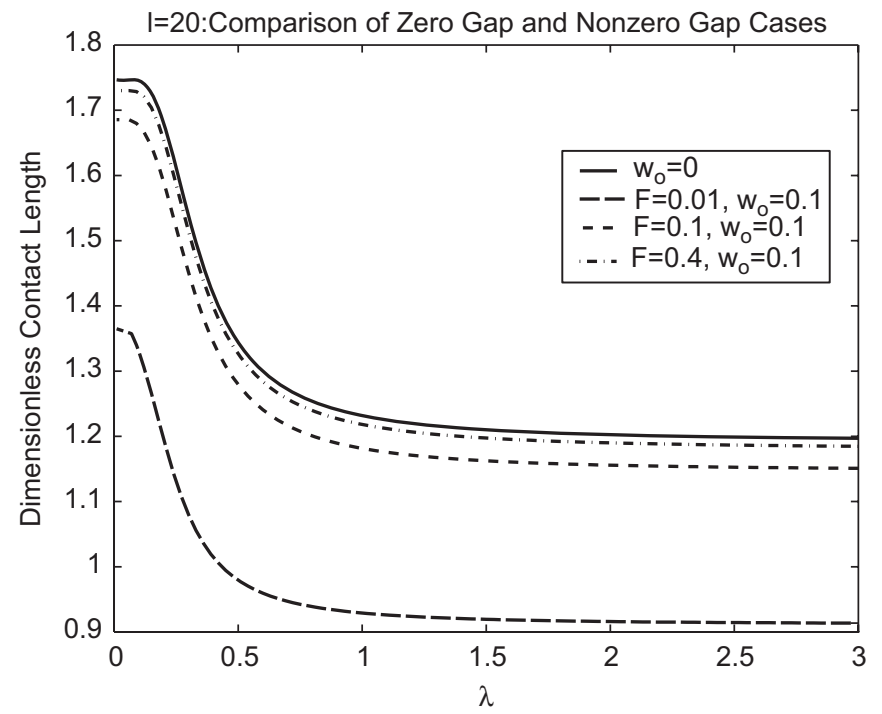

Fig. 6. $\lambda$ versus contact length of a hinged-hinged beam of $l=20$ and $w_{o}=0.1$ under different symmetric loadings and their comparison with $l=20$ and $w_{0}=0$ case.

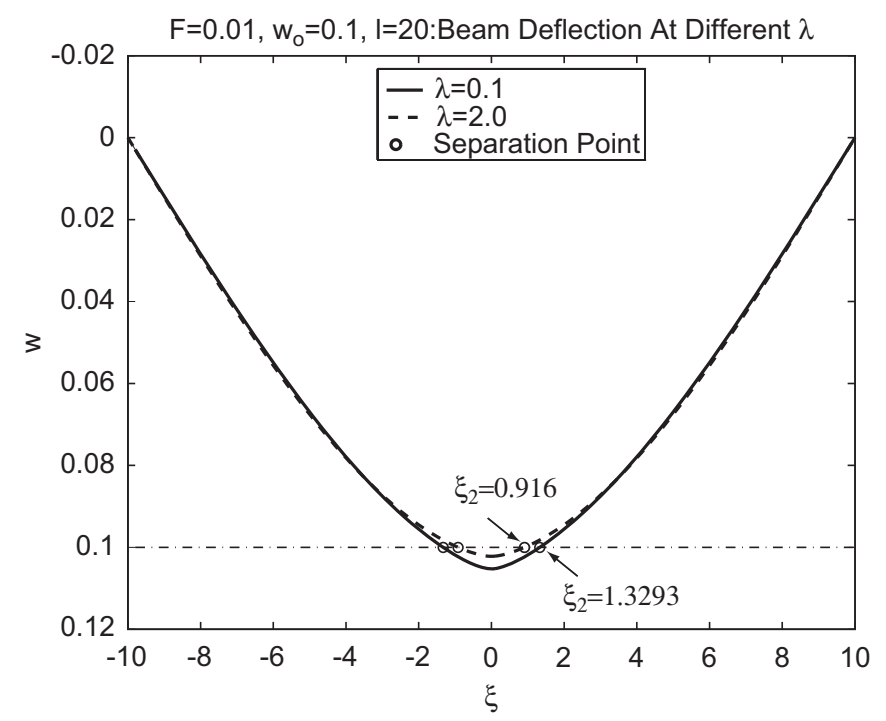

Fig. 7. The deflections of the beam with $l=20, w_{o}=0.1, \lambda=0.1$ and 2.0 under a smaller symmetric load of $F=0.01$.

the beam with $l=20, w_{0}=0.1$ under a much larger load $F=0.4$ for $\lambda=0.1$ and 2 . The contact length now is $\xi_{1}=\xi_{2}=1.737$ when $\lambda=0.1$ and $\xi_{1}=\xi_{2}=1.19$ when $\lambda=2$. The deflections in Figs. 7 and 8 are dramatically different from one another no matter what $\lambda$ value is. Figs. 7 and 8 demonstrate that for the finite beam with nonzero gap distance the contact length is dependent on the magnitude of $F$. Here it will be more reasonable to use $F / w_{o}$ rather than $F$ as a parameter to evaluate the contact. When $w_{o}=0$, no matter how small $F$ is, $F / w_{0} \rightarrow \infty$ and this explains why the contact length of beam with zero gap distance is independent of the loading magnitude. For the beam with a fixed nonzero $w_{0}$, $F / w_{0}$ becomes larger and larger with the increase of $F$. This can be used to explain why in Fig. 6 those curves of $w_{0}=0.1$ approach that of $w_{0}=0$ with $F$ increasing.

A hinged-hinged beam is used here as the study case. Other types of beam end boundary conditions such as free-free, clamped-clamped cantilever ones, etc., can also be studied with the change of Eq. (18).

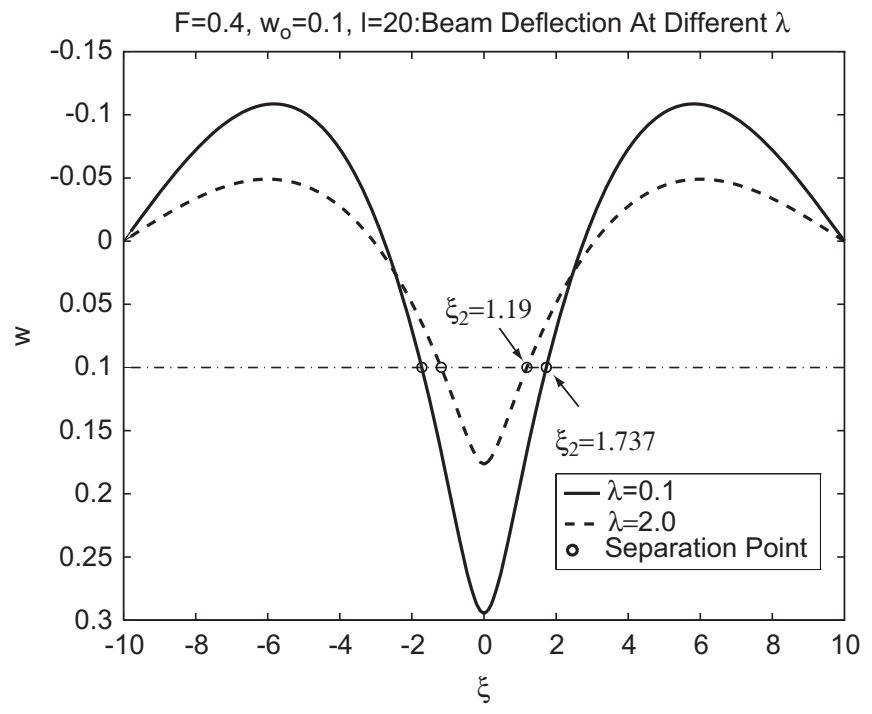

Fig. 8. The deflections of the beam with $l=20, w_{0}=0.1, \lambda=0.1$ and 2.0 under a larger symmetric load of $F=0.4$.

\section{Conclusion}

The contact lengths and deflections of a finite beam with zero/ nonzero gap distance, different lengths and symmetric/asymmetric loading on a Reissner foundation are discussed. The beam length and gap distance are vital on determining the contact length. For a relatively short beam, the loading location is also very important and the asymmetric loading results in the difference of left and right contact lengths. When the gap distance is zero and the beam length becomes larger and larger, the difference between the symmetric and asymmetric loadings becomes smaller and smaller; the contact length approaches the one obtained by Weitsman for an infinitely long beam. When the gap distance is zero, the contact length of a finite beam is again demonstrated to be independent of the load magnitude as that of an infinitely long beam. But that of a finite beam with nonzero gap distance is demonstrated to be dependent on the load magnitude. Therefore, $F / w_{0}$ is proposed as a parameter to evaluate the contact length. For the fixed nonzero gap distance case, the beam contact length approaches that of the zero gap distance case when $F$ approaches infinity (so is $F / w_{o}$ ).

\section{Acknowledgments}

This work is supported by both the National Natural Science Foundation of China (NSFC, Grant nos. 10502050 and 10721202) and the Scientific Research Foundation for the Returned Overseas Chinese Scholars, State Education Ministry of China.

\section{References}

[1] Timoshenko SP, Woinowsky-Krieger S. Theory of plates and shells. 2nd ed. New York, NY: McGraw-Hill Book Company Inc.; 1959.

[2] Weitsman Y. On the unbonded contact between plates and an elastic half space. Journal of Applied Mechanics 1969;36(2):505-9.

[3] Weitsman Y. On foundations that reacts in compression only. Journal of Applied Mechanics 1970;37(7):1019-30.

[4] Lin L, Adams GG. Beam on tensionless elastic foundation. Journal of the Engineering Mechanics Division-ASCE 1987;113(4):542-53.

[5] Li H, Dempsey JP. Unbonded contact of square plate on an elastic half-space or a Winkler foundation. Journal of Applied Mechanics 1988;55:430-6.

[6] Celep Z. Rectangular plates resting on tensionless elastic foundation. Journal of the Engineering Mechanics Division-ASCE 1988;114(12):2083-92. 
[7] Celep Z, Turhan D, Al-Zaid RZ. Contact between a circular plate and tensionless edge support. International Journal of Mechanical Sciences 1988;30(10):733-41.

[8] Akbarov SD, Kocatürk K. On the bending problems of anisotropic (orthotropic) plates resting on elastic foundations that react in compression only. International Journal of Solids and Structures 1997;34:3673-89.

[9] Silva A, Silveira R, Gonclaves P. Numerical methods for analysis of plates on tensionless elastic foundations. International Journal of Solids and Structures 2001;38:2083-100.

[10] Zhang Y, Murphy KD. Response of a finite beam in contact with a tensionless foundation under symmetric and asymmetric loading. International Journal of Solids and Structures 2004;41:6745-58.

[11] Kerr AD. Elastic and viscoelastic foundation models. Journal of Applied Mechanics 1964;31:491-8.

[12] Tsai NC, Westmann RE. Beams on tensionless foundation. Journal of the Engineering Mechanics Division-ASCE 1967;93:1-12.

[13] Dempsey JP, Keer LM, Patel NB, Glasser ML. Contact between plates and unilateral supports. Journal of Applied Mechanics 1984;55:324-8.

[14] Dempsey JP, Li H. Rectangular plates on unilateral edge supports: part 1-theory and numerical analysis. Journal of Applied Mechanics 1986;53:146-50.

[15] Kerr AD. On the unbonded contact between elastic and elastic-rigid media. Acta Mechanica 1979;33:135-46.
[16] Hu C, Hartley GA. Analysis of a thin plate on an elastic half-space. Computers \& Structures 1994;52:227-35.

[17] Johnson KL. Contact mechanics. Cambridge, UK: Cambridge University Press; 1985.

[18] Kerr AD. On the formal development of elastic foundation models. IngenieurArchiv 1984;54:455-64.

[19] Vlasov VZ, Leontiev NN. Beams, plates and shells on elastic foundations. Translated from Russian by Israel Program for Scientific Translations, NTIS Accession No. N67-14238.

[20] Reissner E. A note on deflection of plates on a viscoelastic foundation. Journal of Applied Mechanics 1958;25:144-5.

[21] Girja Vallabhan CV, Das YC. Parametric study of beams on elastic foundations. Journal of the Engineering Mechanics Division-ASCE 1988;113(4):542-53.

[22] Mastrangelo $\mathrm{CH}$, Hsu $\mathrm{CH}$. Mechanical stability and adhesion of microstructures under capillary forces: II. Experiments. Journal of Microelectromechanical Systems 1993;2:44-55.

[23] Yang FQ. Contact deformation of a micromechanical structure. Journal of Micromechanics and Microengineering 2004;14:263-8.

[24] Zhang Y, Zhao Y. Vibration of an adhered microbeam under a periodically shaking electrical force. Journal of Adhesion Science and Technology 2005;19(9):799-815

[25] Korn GA, Korn TM. Mathematical handbook for scientists and engineers. 2nd ed. New York, NY: McGraw-Hill Book Company; 1968. 